\title{
Video-Rate Photometric Stereo-Imaging with General Lighting Luminaires
}

\author{
Johannes Herrnsdorf ${ }^{1}$, Laurence Broadbent ${ }^{2}$, Glynn C. Wright ${ }^{2}$, Martin D. Dawson ${ }^{1}$, and Michael J. Strain ${ }^{1}$ \\ ${ }^{1}$ Institute of Photonics, Department of Physics, University of Strathclyde, Glasgow G1 1RD (UK), \\ ${ }^{2}$ Aralia Systems, Bristol Robotics Laboratory, Bristol BS16 1QY (UK), \\ Email: johannes.herrnsdorf@strath.ac.uk
}

\begin{abstract}
D images of moving objects can be achieved with a surveillance camera and four white light-emitting diodes. With these simple components, an imaging rate of $15 \mathrm{~Hz}$ is possible, limited by the camera framerate.
\end{abstract}

\section{INTRODUCTION}

Photometric stereo-imaging is a $3 \mathrm{D}$ imaging technique that can provide the surface normal vectors and surface albedo of the imaged objects. It relies on illumination of the scene from different angles and computational 3D surface reconstruction by assuming a Lambertian reflectance. Sophisticated photometric stereo-imaging setups have been devised for high resolution 3D imaging of facial expressions and the movement of clothes [1], [2]. These demonstrations employed light-projectors, multiple cameras, and combined photometric stereo-imaging with other 3D imaging aproaches. In studio environments, 3D imaging rates of $17 \mathrm{~Hz}$ with conventional photometric stereo [3] and $60 \mathrm{~Hz}$ with multispectral photometric stereo [2] were achieved.

Capitalizing on advances in both digital cameras and gallium nitride based light-emitting diodes (LEDs) for general lighting, simpler photometric stereo-imaging setups have been developed in the recent past [4], [5]. These systems employ a single camera and a small number (4-7) of LEDs. Therefore, they are suitable for wider deployment than the previous systems, making them attractive for portable face-recognition systems [4] or imaging robots [5]. However, these systems operated at an update rate of about $1 \mathrm{~Hz}$, which is not adequate for $3 \mathrm{D}$ imaging of scenes with moving objects.

Here, we report photometric stereo-imaging using a surveillance camera and four high-brightness LEDs with a full 3D update rate of $15 \mathrm{~Hz}$, limited by the camera frame rate of $60 \mathrm{fps}$. Successful reconstruction of the surface normals of a moving object is demonstrated. Our setup is well-suited for monitoring room-scale scenes e.g. in manufacturing sites, hospitals, or security-sensitive areas. Crucially, the high camera frame rate reduces the perceived visual flicker and thus enables utilization of the room-lighting for the purpose of $3 \mathrm{D}$ imaging.

\section{EXPERIMENT}

As illustrated in figure 1, four LEDs were placed around the camera (Point Grey Flea 3) in an X-shaped geometry. The separation $d$ between the LEDs was about $30 \mathrm{~cm}$ and the imaging distance $L$ was $0.5 \mathrm{~m}$. The camera captured frames with a resolution of $640 \times 480$ at a rate of $60 \mathrm{fps}$. The LEDs

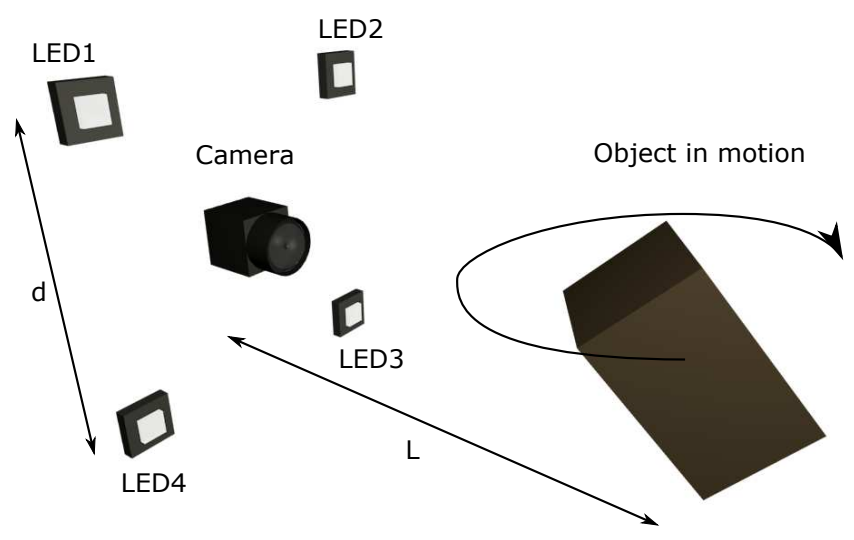

Fig. 1. Schematic of the experimental setup.

TABLE I

CHARACTERISTICS OF THE LEDS IN EMITTER-FOLLOWER CONFIGURATION.

\begin{tabular}{|l||c|c|c|c|}
\hline & Red & Green & Blue & White \\
\hline$I_{L E D}$ at $V_{B}=3.3 \mathrm{~V}[\mathrm{~mA}]$ & 1000 & 93 & 132 & 127 \\
$V_{B}$ at $I_{L E D}=0.7 \mathrm{~A}[\mathrm{~V}]$ & 3.01 & 4.07 & 4.16 & 4.14 \\
Bandwidth $[\mathrm{MHz}]$ & 3.3 & 13.6 & 15.9 & 8.6 \\
\hline
\end{tabular}

were synchronized with the camera and a full 3D update of the scene is obtained every four frames.

As luminaires, we used high brightness red-green-bluewhite LEDs (Osram OSTAR Stage LE RTDUW S2W) with a nominal operating current of $0.7 \mathrm{~A}$ for each of the four LED elements on the chip. At this current, the white LED element emits a luminous flux of $200 \mathrm{~lm}$. Each LED was driven by an $n p n$ power transistor (BCX54) in emitter-follower configuration. The transistor base acts as a high impedance voltage input $V_{B}$ that allows to address the LED elements with voltage outputs from logic circuits with low current driving capability. Table I gives the dependence between $V_{B}$ and the LED current $I_{L E D}$ at the nominal driving current of $0.7 \mathrm{~A}$ and also at an input voltage of $3.3 \mathrm{~V}$, corresponding to a common logic voltage level. The table also gives the $-3 \mathrm{~dB}$ electrical-to-optical bandwidth of the LEDs when a 0-3.3 V square wave was applied to $V_{B}$. Despite the high modulation depth, the bandwidths of 3-16 $\mathrm{MHz}$ are of comparable order of magnitude as the small-signal bandwidth of typical highpower LEDs [6]. 
a)

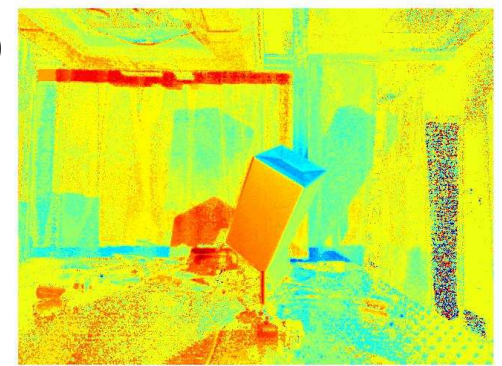

$\mathrm{nx}$

d)

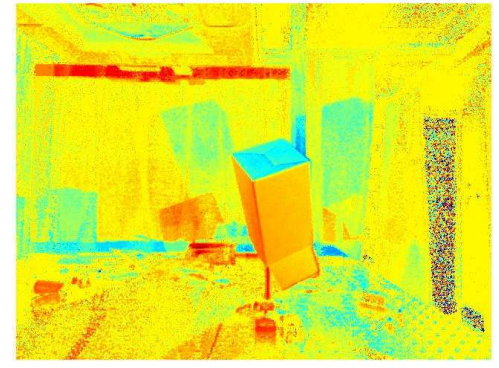

b)

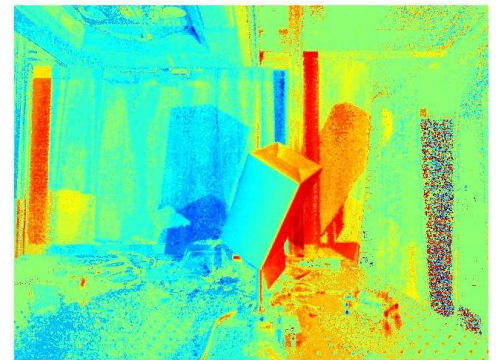

ny

e)

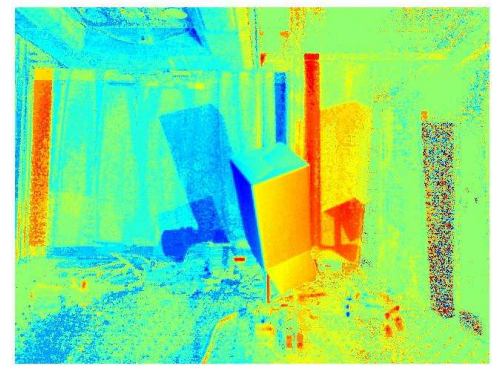

c)

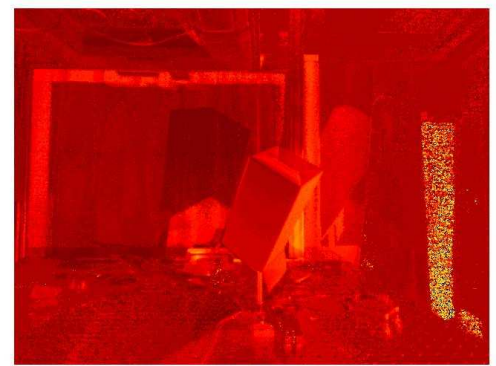

$\mathrm{nz}$

f)

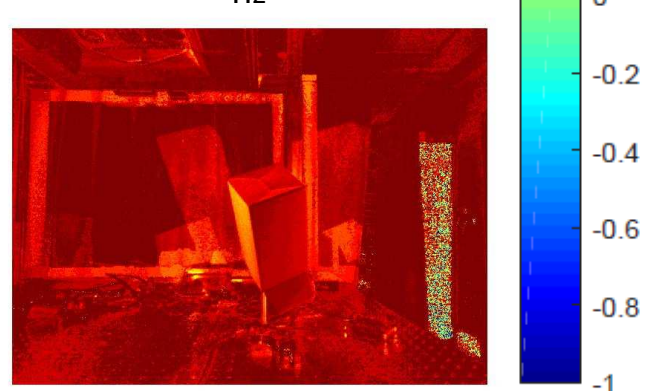

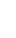

Fig. 2. Components of the surface normal vector $\vec{n}$ at times $t_{1}$ and $t_{2}$, where $t_{2}$ was 1.13 seconds after $\left.t_{1}: a\right) n_{x}$ at $\left.t_{1}, b\right) n_{y}$ at $t_{1}, c$ ) $n_{z}$ at $\left.t_{1}, d\right) n_{x}$ at $\left.t_{2}, e\right) n_{y}$ at $t_{2}$, and $\left.f\right) n_{z}$ at $t_{2}$.

To demonstrate imaging of a moving object, a white cardboard box was placed in the scene. A stepper motor was used to rotate the box at $6 \mathrm{RPM}$, i.e. it took $10 \mathrm{~s}$ for the box to revolve once around itself.

\section{RESULTS}

Photometric stereo-imaging allows determination of the surface normal vectors $\vec{n}$ of the imaged object at each pixel in the captured frame. A representative set of surface normals obtained in the setup is shown in figures $2 \mathrm{a}-\mathrm{c}$. It can clearly be seen how the $n_{x}$ component distinguishes between up and down facing sides of the box, and $n_{y}$ distinguishes between left and right facing sides. The out-of-plane component $n_{z}$ is always positive, i.e. points towards the camera, because surfaces with negative $n_{z}$ are hidden from view. However, its magnitude still varies visibly according to the direction of the face.

Note that these surface normals were obtained while the box was in motion. Despite this, the boundaries of the box are sharp and well-defined, proving that the imaging rate is adequate to resolve the motion. The box was constantly imaged while moving and a second representative set of surface normals is shown in figures $2 \mathrm{~d}-\mathrm{f}$. The position of the box has changed between the two sets, and it can be seen how the change in the 3D orientation of the sides of the box is reflected in the values of the components of $\vec{n}$.

\section{DISCUSSION AND CONCLUSION}

The results show that, by using simple low-cost components, 3D imaging is possible at rates that are sufficient to resolve motion comparable to that of persons moving in a room.
The setup dimensions and imaging distance here were chosen to fit the experiment on an optical table. However, there is no hindrance to extend the dimensions to room-scale, as the type of LED used here is already widely employed for general indoors lighting and cameras of this type are used in surveillance applications. This work opens opportunities to use LED-based indoors lighting for imaging purposes.

\section{ACKNOWLEDGMENT}

The authors thank the Engineering and Physical Sciences Research Council for funding under Partnership Resource of QuantIC, grant number EP/M01326X/1. Data are available online at https://pure.strath.ac.uk/portal/.

\section{REFERENCES}

[1] L. Zhang, N. Snavely, B. Curless, and S. M. Seitz, Spacetime Faces: High-Resolution Capture for Modeling and Animation. London: Springer London, 2008, pp. 248-276.

[2] C. Hernandez, G. Vogiatzis, G. J. Brostow, B. Stenger, and R. Cipolla, "Non-rigid photometric stereo with colored lights," in 2007 IEEE 11th International Conference on Computer Vision, Oct 2007, pp. 1-8.

[3] T. Weise, B. Leibe, and L. V. Gool, "Fast 3d scanning with automatic motion compensation," in 2007 IEEE Conference on Computer Vision and Pattern Recognition, June 2007, pp. 1-8.

[4] Y. Zhang, G. M. Gibson, R. Hay, R. W. Bowman, M. J. Padgett, and M. P. Edgar, "A fast 3D reconstruction system with a low-cost camera accessory," SCIENTIFIC REPORTS, vol. 5, JUN 92015.

[5] A. F. Martins, M. Bessant, L. Manukyan, and M. C. Milinkovitch, "(ROBBIE)-O-2-3D, a Fast Robotic High-Resolution System for Quantitative Phenotyping of Surface Geometry and Colour-Texture," PLOS ONE, vol. 10, no. 6, JUN 32015.

[6] J. Grubor, S. Randel, K. D. Langer, and J. W. Walewski, "Broadband information broadcasting using led-based interior lighting," Journal of Lightwave Technology, vol. 26, no. 24, pp. 3883-3892, Dec 2008. 